\title{
Fluorescence spectral studies on interaction of SEGS with BSA
}

\author{
Fuchun $\mathrm{Si}^{1}$, Wenbin Wang ${ }^{1,2, a}$, Xuejie Song ${ }^{1}$, Xin Zhao ${ }^{1}$ and Lulu Hao ${ }^{1}$ \\ ${ }^{1}$ Laboratory of molecular biology for TCM, Henan University of Chinese Medicine, Zhengzhou 450046, China; \\ ${ }^{2}$ National Key Laboratory of Cryospheric Sciences/Tien Shan Glaciological Station, Cold and Arid Regions \\ Environmental and Engineering Research Institute, Chinese Academy of Sciences, Lanzhou 730000, China
}

\begin{abstract}
The seeds of Gleditsia sinensis is one of the most effective drugs for the treatment of esophageal carcinoma. To definite the active ingredients of the seeds of gleditsia sinensis, SEGS was extracted from the seeds of Gleditsia sinensis. Investigations the interaction between the active ingredient of traditional Chinese medicine and BSA is helpful for explaining the medicine mechanism. Under the simulative human physiological conditions, the interaction between SEGS and bovine serum albumin (BSA) was investigated by fluorescence spectroscopy. The quenching mechanism of SEGS with BSA is dynamic quenching, SEGS bound with BSA by van der Waals forces or hydrogen bond. The binding constants and the number of binding sites between SEGS and BSA at different temperatures (298 and $308 \mathrm{~K}$ ) were obtained.
\end{abstract}

\section{Introduction}

Bovine Serum Albumin (BSA) is the most abundant soluble protein in the blood plasma, and can help to keep colloidal osmotic blood pressure and act as the transportation and disposition of many drugs, and is capable of binding, delivering an extraordinarily diverse range of endogenous and exogenous compounds like fatty acids, nutrients, steroids, certain metal ions, hormones, enzymes, surfactants and a variety of therapeutic drugs, through the bloodstream to their target organs[1]. Therefore, BSA plays an important role in the transportation, distribution, free concentration, excretion, metabolism and interaction with the target tissues of these ligands[2]. Investigations the interaction between the active ingredient of traditional Chinese medicine and BSA is helpful for explaining the medicine mechanism. It is especially useful for select anti-medicine vitro, and can giving theory guide for anti-medicine design[3].

Gleditsia sinensis is a widely used traditional Chinese herb that belongs to the Caesalpinioideae family[4]. For the past 10 years, our group have carried out purposeful studies of anti-esophageal

\footnotetext{
${ }^{a}$ Corresponding author: contributepaper@126.com
}

This research was supported by the special grade of the financial support from the China Postdoctoral Science Foundation (Grant No.2016T90669), the National Science Foundation for Post-doctoral Scientists of China (Grant No.2015M582189), the National Science Foundation (Grant No.81550014), the Henan Postdoctoral Sustentation Fund, China (Grant No. 00104256), Jinshui District Science and Technology Bureau Social Program (Grant No. 20132602). 
carcinoma medicine from over 2000 kinds Chinese traditional herbs. The seeds of Gleditsia sinensis (Chinese honey locust) is one of the most effective drugs for the treatment of esophageal carcinoma[5]. The seeds of Gleditsia sinensis were performed extraction by $95 \%$ ethanol. The crude extract was dried and suspended in water and successively treated with chloroform, ethyl acetate and n-butanol. The n-butanol fraction showed good cytotoxic activity to the esophageal carcinoma cells, Eca109, Ec9706 and TE1, by MTT assay. And then the n-butanol fraction subjected to Dianion HP-20 column and successively eluted with $\mathrm{H}_{2} \mathrm{O}, 30 \%, 50 \%, 70 \%, 90 \%(\mathrm{v} / \mathrm{v}) \mathrm{EtOH}-\mathrm{H}_{2} \mathrm{O}$, respectively. The $70 \%$ EtOH fraction was chromatographed on silica gel. Sub-fraction was subjected to repeating column chromatography on silica geland further purified with recrystallization, obtained colourless power(SEGS).

\section{Experimental}

\subsection{Apparatus and Reagents}

F-7000 fluorescence spectrophotometer (Hitachi, Japan), pHS-3C digital pH-meter (Shanghai REX Instrument Corp., China) Bovine serum albumin, BSA (Beijing Olympic nemesis Biological Technology Co. Ltd.China, relative molecular mass 65000), was dissolved daily in $0.1 \mathrm{~mol} / \mathrm{L}$ Tris$\mathrm{HCl}$ buffer solution $(\mathrm{pH}=7.3)$ to prepare a solution $\left(1 \times 10^{-5} \mathrm{~mol} / \mathrm{L}\right)$, SEGS to prepare a solution $(80 \mathrm{mg} / \mathrm{L})$, All chemicals were analytical-reagent grade. All solutions were prepared with doubledistilled water.

\subsection{Methods}

Fluorescence spectra were carried out on a Hitachi F-7000 fluorescence spectrophotometer using 1.0 $\mathrm{cm}$ quartz cells. The excitation wavelength of BSA studied in this work was $280 \mathrm{~nm}$.

\section{Results and discussion}

\subsection{Fluorescence quenching of BSA}

Fluorescence technique is wide spread application in investigating of interactions between the active ingredient of traditional Chinese medicine and protein molecules[6]. It is well known that BSA has intrinsic fluorescence due to the presence of amino acids, mainly tryptophan (Trp) and ty rosine (Tyr) residues. The interaction between SEGS and BSA has been mornitored upon exciting BSA at $280 \mathrm{~nm}$ where both Trp and Tyr residues get excited. The variations of emission spectra was shown in Figure 1. The fluorescence quenching spectra of solutions containing a BSA fixed concentration and different concentrations of SEGS at $298 \mathrm{~K}$ and $308 \mathrm{~K}$, It can be observed that the fluorescence intensity of BSA decreases regularly with the increase addition of SEGS, under the experimental condition, the SEGS does not produce any emission in the specified range of study, and there is no significant emission wavelength shift. These results suggest that SEGS interact with BSA can quench BSA intrinsic fluorescence. 

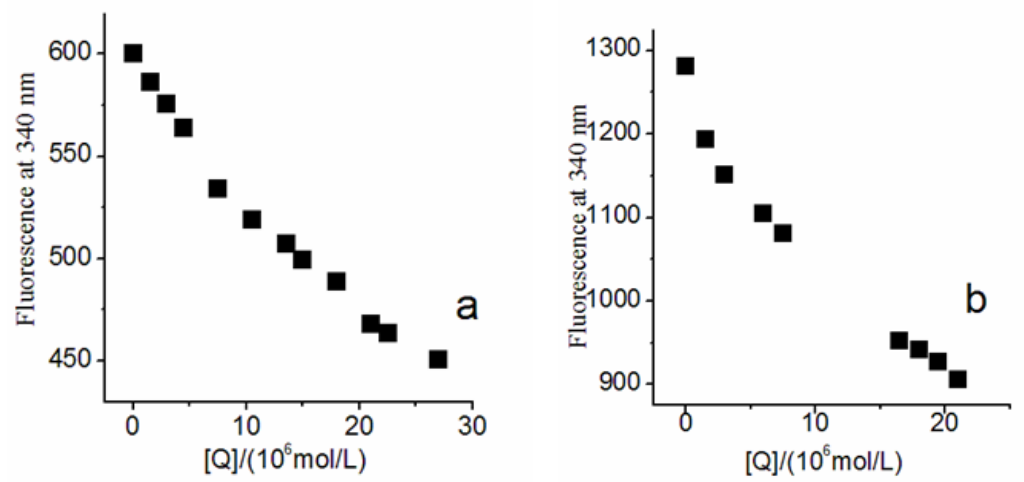

Figure 1. Changes in the emission spectra $(\lambda \mathrm{ex}=280 \mathrm{~nm})$ of the BSA $\left(1.0 \times 10^{-5} \mathrm{~mol} / \mathrm{L}\right)$ upon increasing concentrations of SEGS (a) $298 \mathrm{~K}$ and (b) $308 \mathrm{~K}$

\subsection{Determination of quenching mechanism}

There are two quenching types in characterizing the mechanism of the interaction between quenchers and macromolecules: static and dynamic quenching. Static quenching refers to the formation of a nonfluorescence fluorophore-quencher complex. With temperature increasing, the stability of fluorophore-quencher complex reduced, the quenching constant decreased. However, opposite feature exists in dynamic quenching, Dynamic quenching refers to the quencher diffusing towards the fluorophore during the lifetime of the excited state and, upon contact, the fluorophore returns to the ground state without emission of a photon. Dynamic quenching mechanism was probed using the Stern-Volmer equation[7]:

$$
F_{0} / F=1+K_{q} \tau_{0}[\mathrm{Q}]=1+K_{\mathrm{sv}}[\mathrm{Q}]
$$

Where $F_{0}$ and $F$ represent the fluorescence intensities of BSA in the absence and in the presence of the external quencher, $K \mathrm{q}$ is the bimolecular quenching rate constant, $K_{\mathrm{SV}}$ is the Stern-Volmer quenching constant, $\tau_{0}$ is the average lifetime of biopolymers without quencher, the fluorescence lifetime of the biopolymers is $10^{-8} \mathrm{~s}[8],[\mathrm{Q}]$ is the concentration of the quencher.

This work studied the fluorescence spectrum of the action between BSA and SEGS, obtained the plot of $F_{0} / F$ against [Q] at different temperature shown in Figure 2.
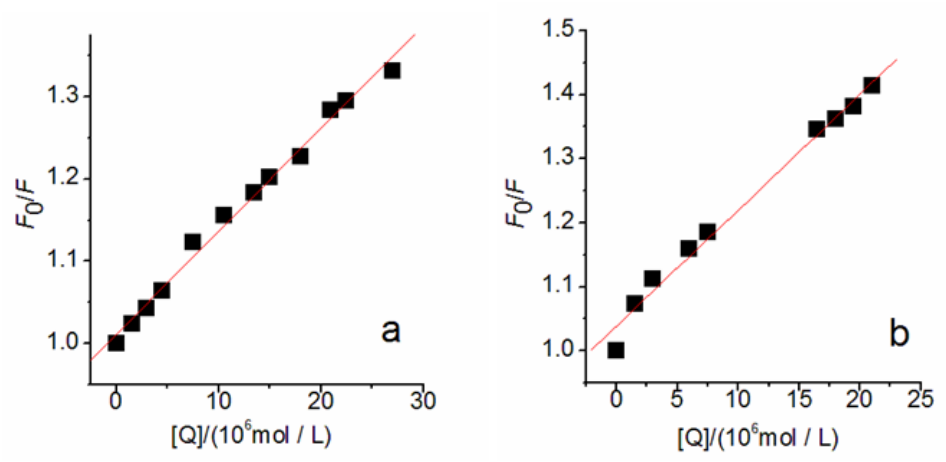

Figure 2. Stern-Volmer plot for the quenching of BSA by SEGS at different temperatures (a) $298 \mathrm{~K}$ and (b) $308 \mathrm{~K}$ 
Equation (1) was applied to determine $K_{\mathrm{SV}}$ by linear regression from a plot of $F_{0} / F$ against [Q], hence, $K \mathrm{q}$ can be caculate by $K \mathrm{q}=K_{\mathrm{SV}} / \tau_{0}$. As shown in Table 1 .

Table 1. Constant of the systems of interaction between SEGS and BSA at different temperatures

\begin{tabular}{ccc}
\hline $\mathrm{T} / \mathrm{K}$ & $\mathrm{Ksv} /(\mathrm{L} / \mathrm{mol})$ & $\mathrm{Kq} /(\mathrm{L} / \mathrm{mol} \cdot \mathrm{s})$ \\
\hline 298 & $1.25 \times 10^{-2}$ & $1.25 \times 10^{6}$ \\
\hline 308 & $1.81 \times 10^{-2}$ & $1.81 \times 10^{6}$ \\
\hline
\end{tabular}

For the SEGS-BSA systems, the values of $K_{\mathrm{Sv}}$ and $K \mathrm{q}=K_{\mathrm{SV}} / \tau_{0}$ obtained from the plots at $298 \mathrm{~K}$ and $308 \mathrm{~K}$ are shown in Table 1. According to the literatures[9-10], which suggest that the fluorescence quenching mechanism is mainly dynamic quenching.

\subsection{The binding constant $K b$ and the number of binding sites $n$}

The relationship between fluorescence quenching intensity and the concentration of quenchers can be used to obtain the binding constant and the number of binding sites[11].

$$
\operatorname{Lg}\left(F_{0}-F\right) / F=\operatorname{Lg} K_{b}+\mathrm{nLg}[\mathrm{Q}]
$$

The values of $K_{\mathrm{b}}$ and $\mathrm{n}$ at $298 \mathrm{~K}$ and $308 \mathrm{~K}$ were obtained from the double logarithm regression curve (Figure 3).

The values of $K_{\mathrm{b}}$ and $\mathrm{n}$ at $298 \mathrm{~K}$ and $308 \mathrm{~K}$ are listed in Table 2.
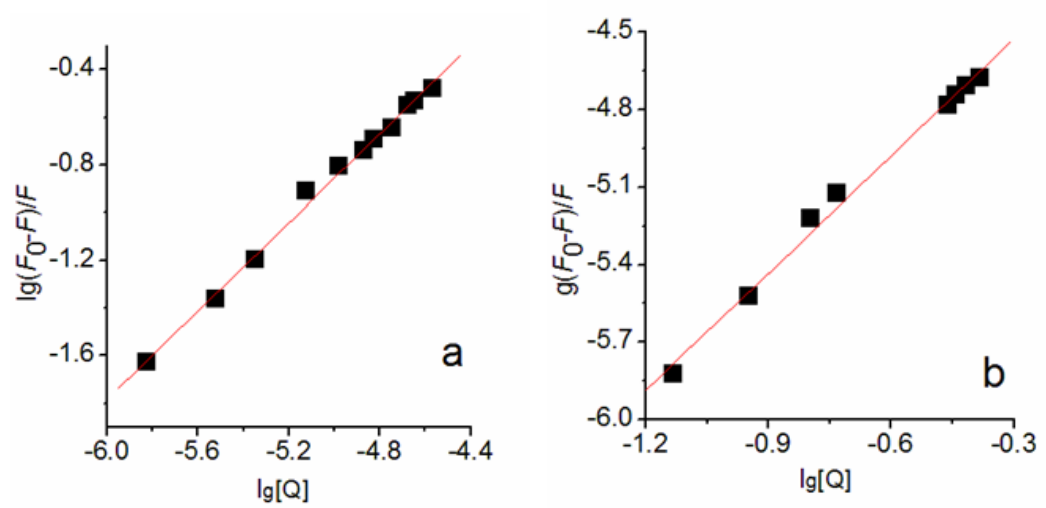

Figure 3. The plots of $\lg \left(F_{0^{-}}-F\right) / F$ vs. $\lg [\mathrm{Q}]$ at different temperatures (a) $298 \mathrm{~K}$ and (b) $308 \mathrm{~K}$ The values of $K_{\mathrm{b}}$ and $\mathrm{n}$ at $298 \mathrm{~K}$ and $308 \mathrm{~K}$ are listed in Table 2.

Table 2. The binding constant $K_{\mathrm{b}}$, and the number of binding sites $\mathrm{n}$

\begin{tabular}{ccccc}
\hline $\mathrm{T} / \mathrm{K}$ & double logarithm regression curve & $\mathrm{r}$ & $k_{\mathrm{b}}$ & $\mathrm{n}$ \\
\hline 298 & $\lg \left(F_{0}-F\right) / F=3.7811+0.9273 \lg [\mathrm{Q}]$ & 0.99746 & $6.041 \times 10^{2}$ & 0.9273 \\
308 & $\lg \left(F_{0}-F\right) / F=2.6680+0.6565 \lg [\mathrm{Q}]$ & 0.99648 & $4.656 \times 10^{2}$ & 0.6565
\end{tabular}

The $\mathrm{n}$ values are nearly 1 , and thus indicate the existence of a single binding site in BSA for SEGS. The interactions of SEGS with BSA decreased when rising the temperature, which suggest that interactions of SEGS with BSA were exothermic[12]. 


\subsection{Binding modes}

The acting forces between drug and biomolecule are composed of weak interactions of molecules such as hydrogen bond formation, van der Waals forces, electrostatic forces, and hydrophobic interaction. The thermodynamic parameters could be calculated by equation (3), (4) and (5).

$$
\begin{gathered}
\operatorname{Ln}\left(K_{2} / K_{l}\right)=\Delta \mathrm{rHm}(1 / \mathrm{T} 1-1 / \mathrm{T} 2) / \mathrm{R} \\
\Delta \mathrm{rGm}=-\mathrm{RTLnK} \\
\Delta \mathrm{rSm}=(\Delta \mathrm{rHm}-\Delta \mathrm{rGm}) / \mathrm{T}
\end{gathered}
$$

The thermodynamic parameters are presented in Table 3.

Table 3. Thermodynamic parameters for the association of SEGS with BSA

\begin{tabular}{lcll}
\hline $\mathrm{T} / \mathrm{K}$ & $\Delta \mathrm{rHm} /(\mathrm{KJ} / \mathrm{mol})$ & $\Delta \mathrm{rSm} /(\mathrm{J} / \mathrm{K})$ & $\Delta \mathrm{rGm} /(\mathrm{KJ} / \mathrm{mol})$ \\
\hline 298 & -19.78 & -13.42 & -15.87 \\
308 & & -13.44 & -15.73 \\
\hline
\end{tabular}

The negative signs for $\Delta \mathrm{rHm}, \Delta \mathrm{rSm}$ and $\Delta \mathrm{rGm}$ reveal that the binding processes are influenced by temperature, low temperature are spontaneous, high temperature are non-spontaneous. According to the literature $[13,14,15]$, The negative $\Delta \mathrm{H}$ and $\Delta \mathrm{S}$ values suggest the van der Waals forces or hydrogen bond formation exist in SEGS and BSA.

\section{Conclusion}

The interaction between SEGS and BSA has been investigated under simulated physiological

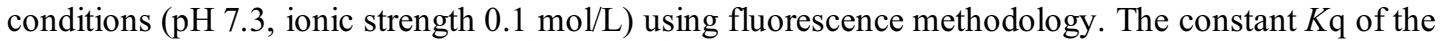
SEGS and BSA is less than $2 \times 10^{10} \mathrm{~L} \cdot \mathrm{mol}^{-1} \cdot \mathrm{s}^{-1}$, which suggest that the fluorescence quenching mechanism of BSA by SEGS was consistent with dynamic quenching. The binding reaction was influenced by temperature for $\Delta \mathrm{rHm}, \Delta \mathrm{rSm}$ and $\Delta \mathrm{rGm}$ is negative. $\Delta \mathrm{H}$ and $\Delta \mathrm{S}$ is negative suggest that Van der Waals forces or hydrogen bond play a major role in the SEGS-BSA interaction.

\section{References}

1. A. Jahanban-Esfahlan, V. Panahi-Azar, Food chemistry, 202 (2016).

2. J. Zhang, H.H. Sun, Y.Z. Zhang, J. Solution Chem, 41 (2012).

3. Y. Li, Y. Gu, H. He, Y. Zhou, Acta Chimica Sinica. 70, 143-150 (2012).

4. D. N. Oleynikov, A. V. Rohin, Applied Biochemistry and microbiology. 46, 103-107(2010).

5. F. C. Si, World Chinese Journal of Digestology. 28, 3139-3145 (2008).

6. R. Suparna, D.Somnath, P. Chiranjit. Spectrochimica acta. Part A, Molecular and biomolecular spectroscopy, 166, 84-94(2016) .

7. X. Zheng, M.P. Ye, Y. Li, Journal of China University of Metrology, 1 (2015).

8. S. Y. Bi, Y. T. Sun, C. Y. Qiao, H. Q. Zhang, C. M. Liu, J. Luminescence.129, 541-547 ( 2009 ).

9. H. Cao, D. Wu, H.Wang, M. Xu, Spectrochim. Acta A 73, 972-975 (2009).

10. F. Cui, Y. Yan, Q. Zhang, X. Yao, G. Qu, Y. Lu, Spectrochim. Acta A 74, 964-971 (2009).

11. L. N. Hordge, K. L. McDaniel, D. D. Jones, et al, Talanta, 152 (2016).

12. F. Y. Zhang, Y. N. Ni, Acta Chimica Sinica. 70, 1379-1384 (2012).

13. F.L. Cui, J.L. Wang, Y.R. Cui, J.P. Li, Anal. Chim. Acta, 571 (2006).

14. N. Nanjundan, P. Selvakumar, R. Narayanasamy, et al. J. Photoch. Photobiolo. B, 141 (2014).

15. L.Wang, D. W. Zhao, L. J. Zi, et al. J. Henan Normal University. 40, 87-90 (2012). 